Aim of the study: To report a pre-suture technique in laparoscopic nephron-sparing surgery (LNSS), which could help reduce and even avoid ischaemia for the treatment of renal cell carcinoma.

Material and methods: Between January and June 2013 we treated 14 patients presenting with renal tumours. The mean age was 46 years and average tumour size was $2.4 \mathrm{~cm}$ in diameter determined by computed tomography (CT). All the patients were treated with LNSS by pre-suturing the resection.

Results: In 13 out of the 14 cases, no clamping was needed during the whole surgery processes, i.e. zero ischaemia was achieved. In the other case, the renal artery was clamped for only 150 seconds due to suture avulsion. The mean operating time was 75 minutes (range 50 to 110 minutes) and mean blood loss was $60 \mathrm{ml}$ (range 30 to $200 \mathrm{ml}$ ). After removal of the drain 2-3 days after surgery, the average postoperative hospital stay time was four days. The surgery had only a minor effect on the renal function. No case of urinary leakage or postoperative bleeding occurred. Postoperative pathological reports showed that the tumours were resected completely with negative surgical margins for all cases. There were no signs of recurrence on follow-up CT performed 1-6 months after surgery.

Conclusions: The pre-suture technique in LNSS reported here required zero or minimal ischaemia time and hence avoided renal ischaemia-reperfusion injury. This surgical technique could be a feasible surgical option for treatment of small, exophytic and peripheral renal tutors.

Key words: renal cell carcinoma, laparoscopic nephron sparing surgery, pre-suture, ischemia.

Contemp Oncol (Pozn) 2014; 18 (5): 355-358 DOI: $10.5114 /$ wo.2014.41385

\section{Zero ischaemia laparoscopic nephron-sparing surgery by re-suturing}

\author{
Jinshan Lu, Qiang Zu, Qingshan Du, Yong Xu, Xu Zhang, Jun Dong
}

Department of Urology, General Hospital of the People's Liberation Army, PR China

\section{Introduction}

Surgery is a standard curative treatment for renal cell carcinoma (RCC). Nephron-sparing surgery for RCC treatment is aimed at maximally maintaining the renal function while achieving a high disease-free survival rate. Laparoscopic nephron-sparing surgery (LNSS) has been accepted widely for the management of RCC [1-4]. After the first case of LNSS [5] showing that this surgical technique had many advantages compared with open surgery, it has evolved remarkably [1]. The earlier ventures that were carried out without controlling the renal vessel by using haemostatic tools (such as diathermy, argon beam coagulation, and ultrasonic shear) $[3,6,7]$, and hence there was no ischaemia time in the surgeries. However, the use of diathermy could lead to positive margin status, and the deep coagulation would result in delayed avascular necrosis as well as urinary fistula formation [1]. The more recent surgical technique that applies renal ischaemia through the clamping of the renal vessels has made it easier to achieve negative margin status. However, the ischaemia-reperfusion injury to the kidney sometimes increases the risk of damaging the renal functions [8]. Although the robot-assisted LNSS surgical technique can reduce the ischaemia time to an acceptable level $[4,9]$, it requires advanced instrumental facilities and considerable expertise, and hence is less feasible for the treatment of RCC in many cases. Here we report a feasible and safe LNSS technique that could reduce the ischaemia time to a minimum, or even to zero, for treatment of small, exophytic, and peripheral renal tumours.

\section{Material and methods}

All the studies herein were in accordance with the Helsinki Declaration of 1975, as revised in 2000, and with the ethical standards of the Local Committee on Human Experimentation. All the patients were aware of and approved this research.

\section{Study population}

Between January and June 2013, 14 patients underwent LNSS. Among the 14 patients, 9 were male and 5 were female. The mean age was 46 years (range 29-67 years). The tumours were on the left in 4 and on the right in 10 patients. In 2 patients the second kidney was suffering from renal calculus, and in the other 12 patients it was healthy. The mean tumour size was $2.4 \mathrm{~cm}$ (range 1.5 to $3.2 \mathrm{~cm}$ ). Computed tomography (CT) (Fig. 1) and Magnetic Resonance Imaging (MRI) were done in all cases, and they were all determined to be stage $\mathrm{T}_{1} \mathrm{~N}_{0} \mathrm{M}_{0}$ renal tumours according to the International Union Against Cancer and the American Joint Committee on Cancer staging system. 


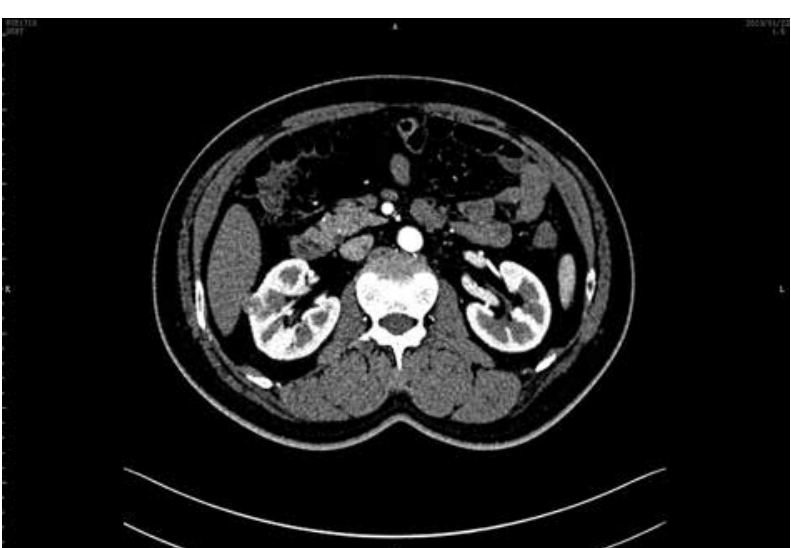

Fig. 1. Preoperative contrast-enhanced computed tomography scan example of renal tumours

\section{Surgical procedure}

\section{Patient position and port distribution}

The patients were placed in the lateral decubitus position, and the waist bridge was heightened. After induction of general anaesthesia, three laparoscopic ports were placed on the lumbar (Fig. 2). The first port (A) was placed below the tip of the twelfth rib. Port B was placed below the subcostal of the anterior axillary line. Port $C$ was placed above the iliac crest, at the mid-axillary line. A 30-degree laparoscope was used in the surgery and $\mathrm{CO}_{2}$ gas was used to create a pneumoperitoneum for working space.

\section{Operation details}

We began the operation by opening the Gerota's fascia via a longitudinal cut, followed by dissection along the renal capsule until the mass was exposed. The fat around the mass was kept in our operations. Then the dissection was taken along the psoas muscle to the dorsal surface of the kidney, such that the renal artery would be exposed sufficiently for one bulldog clamp to be attached if necessary.

Before dissection of the tumour mass, 1-0 absorbable sutures were carried out to pre-suture the kidney parenchyma, by passing the needle forward and back at a distance of $1.5-2 \mathrm{~cm}$ from the tumour margin. The number of passes of the needle was based upon the size of the tumour (generally 2-4 passes), and a Hem-o-lock clip was used to fix one end of suture first. At this point, the suture was not tightened, so that a certain distance from the tumour mass was kept for ease of dissection (Fig. 3A).

Then the tumour mass was dissected from the suturing starting point, in such a way that around $0.5 \mathrm{~cm}$ of normal parenchyma was dissected circumferentially around the mass but without cutting the sutures in the parenchyma (Fig. 3B). Diathermy or an argon beam coagulator was used to keep the bloodless field during the operation if necessary. Along with the dissection, each pre-suture segment was tightened and Hem-o-lock clips were applied to create even tissue tension when each pre-sutur-

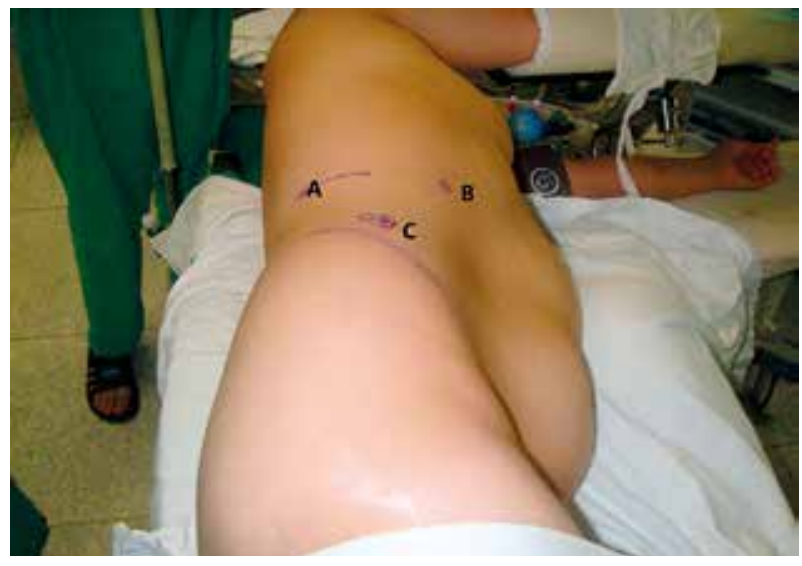

Fig. 2. Patient positioning and laparoscopic port distribution. A, B, and $\mathrm{C}$ were the positions of the three laparoscopic ports.

ing exit point of the parenchyma was reached. Hence, once the tumour mass was removed completely, all the pre-sutures were tightened. Then anther 1-0 absorbable suture was used to seal the cutting surface with the entry, and each exit point was fixed with Hem-o-lock clips, thus eliminating the need to tie knots (Fig. 3 C and D). After confirming that there was no active bleeding, a surgical drain was introduced and the operation was ended with the closure of all the incisions.

\section{Results}

All of the 14 cases were successful, and no conversion to open surgery was necessary. Analyses of the specimens afterwards showed that the tumours and the capsules outside were dissected with $0.5-1.0 \mathrm{~cm}$ margins of healthy parenchyma around them as expected (Fig. 4). Pathological analyses also confirmed that all the 14 cases were RCC, 10 of them were clear cell and four chromophobe RCCs. We achieved negative surgical margins in all 14 cases. The mean operating time was 75 minutes (range 50 to 110 minutes), and the mean blood loss was $60 \mathrm{ml}$ (range 30 to $200 \mathrm{ml}$ ) with no blood transfusion required. In 13 out of the 14 cases, the renal artery was not clamped along the operation and hence zero ischaemia was achieved. For the other case, in which the tumour size was $3.2 \mathrm{~cm}$ in diameter, the renal artery was clamped for only 150 seconds. In this case, one suturing exit point was so close to the edge of the tumour mass that when tightening the suture after the dissection, the suture tore the parenchyma around the resection, resulting in a large amount of bleeding (about $200 \mathrm{ml}$ ) and hence clamping of the renal artery was needed. Another figure-eight suture was applied and the clamping of renal artery was released afterwards. No intraoperative or postoperative organ injuries, pneumothorax, urinary fistula, postoperative bleeding, or secondary infection occurred in any of the 14 cases. The renal functions also remained stable throughout the postoperative period in all cases. The incisions were mildly painful and the drains were removed after 2-3 days. The postoperative hospital stays were 3-4 days. There were no signs of local recurrence or distant metastases on follow-up CT performed 1-6 months after surgeries. 

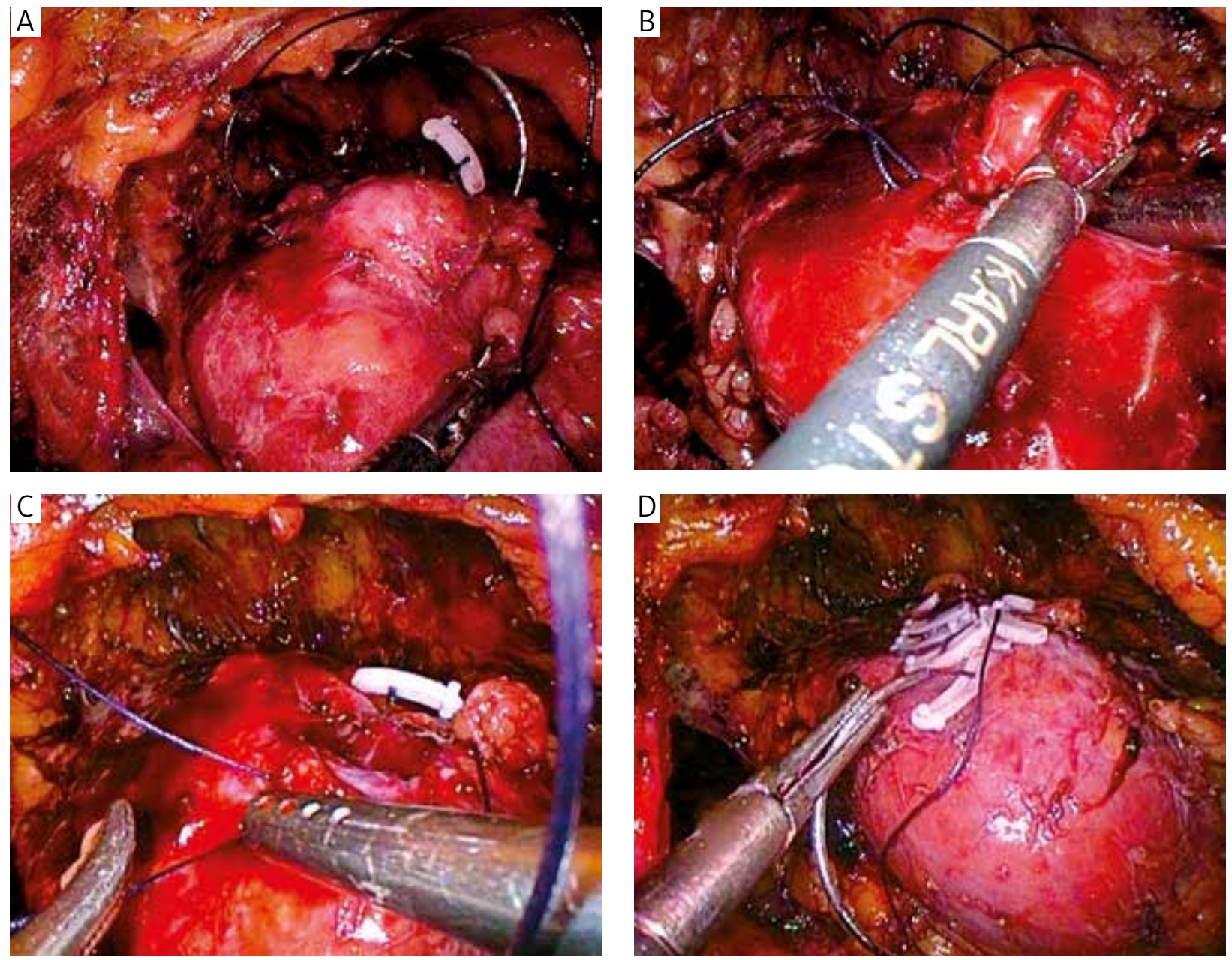

Fig. 3. Operation details. A) Renal artery identification and pre-suturing. B) Renal tumour dissection. C) and D) Tightening the pre-sutures along the dissection and fixing the end with a Hem-o-lock clip

\section{Discussion}

Indications and patient selection

In our experience, this pre-suture LNSS technique is feasible for the treatment of RCCS with small tumour sizes (smaller than $3 \mathrm{~cm}$ in diameter would be optimal) and which are exophytic and peripheral renal tumours.

\section{Technical key points}

The kidney should be mobilised from its surrounding perirenal fat so that the tumour mass would be exposed in a good position for excision. The renal capsule should be kept intact for better closure of the resection. Also, the fat around the tumour mass should be kept to facilitate gripping and traction.

The renal artery should be exposed sufficiently for one bulldog clamp, in case of necessity before excision. In our experience, clamping only the renal artery could achieve a small amount of bleeding. Hence, there is no need to mobilise the renal vein.

If the preoperative diagnostic result indicates malignant tumour, then the tumour mass should be dissected with a $0.5-1.0-\mathrm{cm}$ margin of healthy parenchyma around it. If it is diagnosed to be a hamartoma, then enucleating the renal tumour should be sufficient.
The most essential point of this LNSS technique for the realisation of zero ischaemia is pre-suture before the dissection of the tumour mass. Hence, the control of the positions for suturing entry and exit points as well as the suturing tracks is the key to success for this operation. There should be a $1.5-2.0-\mathrm{cm}$ distance in between the tumour mass with the suturing points. If it is pre-sutured too close to the tumour mass, it would be easy to tear the renal parenchyma edge and thus cause bleeding when tightening the sutures after the excision of the tumour mass. Also, the suturing track should be below the tumour mass to ensure reliable haemostasis and to avoid cutting the sutures during the dissection of the tumour mass. Furthermore, the renal parenchyma is more brittle with blood supply. Hence, the suturing should be performed by advancing the needle along its curvature in order to reduce the risk of damage and bleeding.

The pre-sutures should not be tightened before the excision of the tumour mass. A certain distance should remain between the pre-sutures with the tumour mass for ease of dissection.

A Hem-o-lock clip is fixed to the entry end of the pre-suture. After the excision of the tumour, the sutures are tightened and another Hem-o-lock clip is applied, thus eliminating the need to tie knots. 

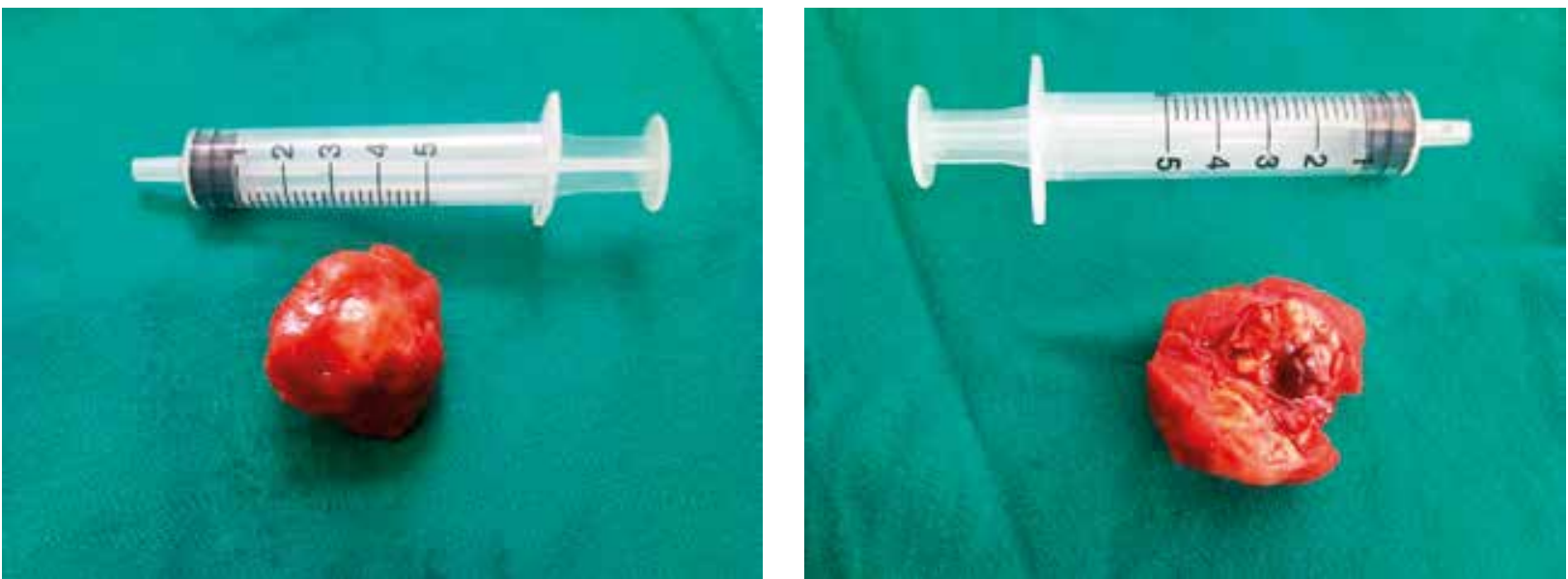

Fig. 4. A fresh LNSS specimen. The tumour mass and the capsules outside were dissected with $0.5-1.0-\mathrm{cm}$ margins of healthy parenchyma circumferentially around them. It was confirmed to be clear cell RCC by pathological analysis

\section{Advantages of this laparoscopic nephron-sparing surgery technique}

The pre-suture technique in LNSS reported here required zero or minimal (only when the suturing point was not well located) ischaemia time and hence avoided renal ischaemia-reperfusion injury. The key point was to pre-suture the resection ahead the excision of the tumour mass. To ensure safety, the renal artery was also mobilised, in case it was necessary for clamping in the event of bleeding. Even if clamping of the renal artery became necessary to controll the bleeding to maintain a bloodless operation field, the time for clamping would be very short compared with traditional LNSS techniques, e.g. one of the 14 cases we studied required only 150 seconds for renal artery clamping.

In summary, pre-suture before dissection of the tumour mass could reduce the ischaemia time or even completely avoid ischaemia, and hence reduce the risk of renal ischaemia-reperfusion injury. This surgical technique could be a feasible surgical option for the treatment of small, exophytic, and peripheral renal tumours. More cases should be studied in future, to improve this LNSS technique such that it would become a feasible treatment for renal tumours of larger size and even for endophytic RCCs.

The authors declare no conflict of interest.

\section{References}

1. Abukora F, Nambirajan T, Albqami N, Leeb K, Jeschke S, Gschwendtner M, Janetschek G. Laparoscopic nephron sparing surgery: evolution in a decade. Eur Urol 2005; 47: 488-93.

2. Gerber GS, Stockton BR. Laparoscopic nephron-sparing surgery. J Endourol 2007; 21: 458-9.

3. Jeschke K, Peschel R, Wakonig J, Schellander L, Bartsch G, Henning K. Laparoscopic nephron-sparing surgery for renal tumors. Urology 2001; 58: 688-92.

4. Laydner H, Autorino R, Spana G, et al. Robot-assisted partial nephrectomy for sporadic ipsilateral multifocal renal tumours. BJU Int 2012; 109: 274-80.

5. Winfield HN, Donovan JF, Godet AS, Clayman RV. Laparoscopic partial nephrectomy: initial case report for benign disease. J Endourol 1993; 7: 521-6.
6. Harmon WJ, Kavoussi LR, Bishoff JT. Laparoscopic nephron-sparing surgery for solid renal masses using the ultrasonic shears. Urology 2000; 56: 754-9.

7. Janetschek G, Jeschke K, Peschel R, Strohmeyer D, Henning K, Bartsch G.. Laparoscopic surgery for stage T1 renal cell carcinoma: radical nephrectomy and wedge resection. Eur Urol 2000; 38: 131-8.

8. Abukora F, Albqami N, Nambirajan T, Ziegerhofer J, Leeb K, Janetschek G. Long-term functional outcome of renal units after laparoscopic nephron-sparing surgery under cold ischemia. J Endourol 2006; 20: 790-3.

9. Masson-Lecomte A, Yates DR, Bensalah K, et al. Robot-assisted laparoscopic nephron sparing surgery for tumors over $4 \mathrm{~cm}$ : Operative results and preliminary oncologic outcomes from a multicentre French study. Eur J Surg Oncol 2013; 39: 799-803.

\section{Address for correspondence}

\section{Jun Dong}

Department of Urology

General Hospital of the People's Liberation Army

28 Fuxing Road

100853 Beijing, China

e-mail: jundong163@126.com

Submitted: 6.08 .2013

Accepted: $\quad 18.02 .2014$ 